the time between symptom onset and physician diagnosis is 5-7 years. Diagnosis delay is attributed to nonspecific presentation of chronic back pain, requiring clinical experience to recognize early AS.

Objectives: To identify obstacles to early diagnosis of AS by assessing the knowledge of nonrheumatology health care providers (HCPs) on inflammatory back pain (IBP) and possible barriers to referral to a rheumatologist.

Methods: Survey content was first conceptualized based on concept elicitation interviews with HCPs, and questionnaires were developed by the study investigators to identify clinical characteristics, symptom presentation, and diagnostic measures that lead an HCP to suspect IBP and their perspectives on the referral process. The survey was then cognitively tested with HCPs from 10 specialties (family medicine, internal medicine, dermatology, gastroenterology, ophthalmology, orthopedics, chiropractic, pain management, physical therapy, and physiatry), revised, and finalized. HCPs from these 10 specialties were invited to participate in the crosssectional web-based survey hosted by Survey Sampling International between June 27 and July 20, 2018. HCPs who were currently licensed, actively practicing in the US, and had referred a patient with suspected IBP (except ophthalmology) or referred a patient with uveitis/iritis (ophthalmology only) within the past 12 months were eligible to participate. Descriptive statistics were used to analyze the data.

Results: Of 2395 HCPs screened, 1690 were eligible and included in our study. Overall, HCPs saw a median of 100 patients with chronic back pain within the past 12 months of the survey. Regarding criteria leading to suspicion of IBP, $61 \%$ of HCPs indicated morning stiffness > 30 minutes, $29 \%$ sleep disturbance due to back pain, $28 \%$ pain that improves with activity, and $16 \%$ alternating buttock pain. Among HCPs who would order diagnostic blood work, $\approx 90 \%$ selected C-reactive protein, erythrocyte sedimentation rate, antinuclear antibody (ANA), and rheumatoid factor (RF), while $76 \%$ selected HLA-B27 (Figure 1).. Most HCPs suspected underlying conditions of rheumatoid arthritis (RA; 94\%), AS $(90 \%)$, and psoriatic arthritis $(81 \%)$ as related to IBP. Almost $40 \%$ of HCPs would treat patients with suspected IBP themselves; of these, $\approx$ $80 \%$ would recommend nonsteroidal anti-inflammatory drugs and physical therapy (Figure 2).. Regarding referrals, 57\% of HCPs would refer patients with suspected IBP to another physician, but only $13 \%$ would refer immediately; $49 \%$ would perform further evaluations, $24 \%$ would wait until initial treatment response, $11 \%$ would monitor patient for some time, and $3 \%$ would consult with another physician before referring. Upon referral, $90 \%$ of HCPs estimated a wait time of up to 2 months for their patient to see another specialist. Of $1670 \mathrm{HCPS}$ queried, $80 \%$ indicated that the specialist's expertise in treating autoimmune disease is the most important factor influencing their referral.

Conclusion: Most HCPs did not recognize IBP criteria in patients with chronic back pain. As most considered RA to be the underlying condition, ANA and RF were ordered as diagnostic workup. Many HCPs opted to treat and monitor patients with suspected IBP before referral, and most reported a wait time of up to 2 months for their patients to see a specialist.

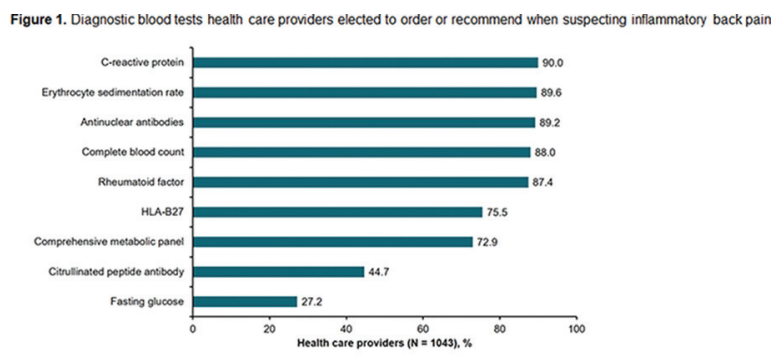

Figure 2. Treatment recommendations by health care providers who opted to treat patients with suspected

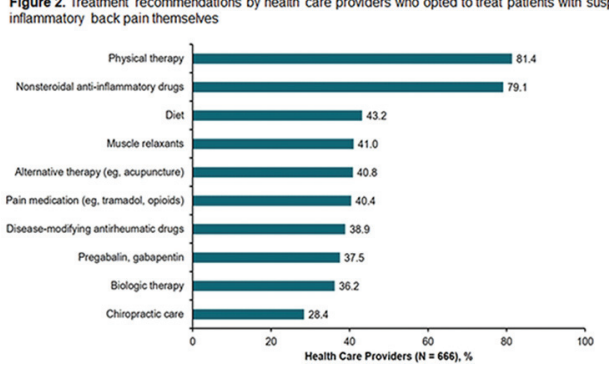

Acknowledgement: This study was sponsored by Novartis Pharmaceuticals Corporation, East Hanover, NJ.

Disclosure of Interests: Marina Magrey Grant/research support from: $M$. Magrey received research funding from AbbVie and UCB for clinical trials, served as a consultant for Novartis, and was a member of advisory boards for Eli Lilly, Novartis, and UCB, Consultant for: M. Magrey received research funding from AbbVie and UCB for clinical trials, served as a consultant for Novartis, and was a member of advisory boards for Eli Lilly, Novartis, and UCB, Esther Yi Consultant for: E. Yi is a postdoctoral fellow at the University of Texas at Austin and Baylor Scott and White Health, providing services to Novartis Pharmaceuticals Corporation., Daniel Wolin Employee of: D. Wolin is an employee of RTI Health Solutions., Mark Price Employee of: M. Price is an employee of RTI Health Solutions., Costel Chirila Employee of: C. Chirila is an employee of RTI Health Solutions., Eric Davenport Employee of: E. Davenport is an employee of RTI Health Solutions., Yujin Park Employee of: Y. Park is an employee of Novartis.

DOI: 10.1136/annrheumdis-2019-eular.501

\section{SAT0339 WHAT IS THE IMPACT OF IMAGING ON DIAGNOSTIC ASCERTAINMENT OF PATIENTS PRESENTING WITH UNDIAGNOSED BACK PAIN AND WHAT IS THE IMPACT OF CENTRAL EVALUATION? DATA FROM THE SCREENING IN AXIAL SPONDYLOARTHRITIS IN PSORIASIS, IRITIS, AND COLITIS (SASPIC) COHORT}

Walter P. Maksymowych ${ }^{1,2}$, Raj Carmona ${ }^{3}$, James Yeung ${ }^{4}$, Jon Chan ${ }^{5}$, Liam Martin $^{6}$, Sibel Aydin ${ }^{7}$, Dianne Mosher ${ }^{6}$, Ariel Masetto ${ }^{8}$, Stephanie Keeling ${ }^{1}$, Olga Ziouzina ${ }^{6}$, Sherry Rohekar ${ }^{9}$, Joel Paschke ${ }^{2}$, Amanda Carapellucci ${ }^{2}$, Robert G. Lambert ${ }^{1} .{ }^{1}$ University of Alberta, Edmonton, Canada; ${ }^{2}$ CaRE Arthritis, Edmonton, Canada; ${ }^{3}$ McMaster University, Hamilton, Canada; ${ }^{4}$ James Yeung Rheumatology, Vancouver, Canada; ${ }^{5}$ Artus Health Center, Vancouver, Canada; ${ }^{6}$ University of Calgary, Calgary, Canada; ${ }^{7}$ University of Ottawa, Ottawa, Canada; ${ }^{8}$ University of Sherbrooke, Sherbrooke, Canada; ${ }^{9}$ Lawson Health Research Institute, London, Canada

Background: Although MRI of the sacroiliac joints (SIJ) is the most sensitive imaging modality for early diagnosis of axial spondyloarthritis $(\operatorname{axSpA})$ it is costly and not readily available. Therefore, clinicians still rely primarily on radiography. The relative degree to which radiography and MRI changes diagnostic ascertainment of axSpA in patients presenting with undiagnosed back pain has not been formally studied.

Objectives: We aimed to assess the relative impact of radiography and MRI evaluation on diagnostic ascertainment of axial SpA in patients presenting with undiagnosed back pain to rheumatologists, and the impact of central evaluation.

Methods: The multicenter Screening for Axial Spondyloarthritis in Psoriasis, Iritis, and Colitis (SASPIC) Study is aimed at early detection of axial $\mathrm{SpA}$. Consecutive patients $\leq 45$ years of age with $\geq 3$ months undiagnosed back pain with any one of psoriasis, acute anterior uveitis (AAU), or colitis undergo routine clinical evaluation by a rheumatologist for axial $\mathrm{SpA}$ and MRI evaluation is ordered per rheumatologist decision. The rheumatologist determines the presence or absence of axial SpA at 3 consecutive stages: 1. After the clinical evaluation; 2. After the results of labs (B27, CRP) and radiography; 3. After the results of MRI evaluation. The eCRF, radiographs, and MRI scans were also assessed centrally. Results: The SASPIC cohort comprises 246 patients $(52.4 \%$ male, mean age 34.5 years, mean symptom duration 7.3 years, mean back pain duration 7.1 years, B27+ 36.2\%) referred with AAU (29.7\%), psoriasis

\begin{tabular}{|c|c|c|c|c|c|}
\hline $\begin{array}{l}\text { Stage of } \\
\text { global } \\
\text { assessment }\end{array}$ & Data source & $\begin{array}{c}\text { axSpA } \\
\text { YES }\end{array}$ & $\begin{array}{c}\text { axSpA YES } \\
\text { with } \\
\text { confidence } \\
>7\end{array}$ & $\begin{array}{c}\text { NOT } \\
\text { axSpA }\end{array}$ & $\begin{array}{c}\text { axSpA NO } \\
\text { with } \\
\text { confidence } \\
<-4\end{array}$ \\
\hline 1. $N=246$ & Clinical & $\begin{array}{c}169 \\
(68.7 \%)\end{array}$ & $50(20.3 \%)$ & $\begin{array}{c}77 \\
(31.3 \%)\end{array}$ & $40(16.3 \%)$ \\
\hline 2. $N=246$ & $\begin{array}{l}\text { Clinical plus } \\
\text { radiography }\end{array}$ & $\begin{array}{c}141 \\
(57.3 \%)\end{array}$ & $66(26.8 \%)$ & $\begin{array}{c}105 \\
(42.7 \%)\end{array}$ & $78(31.7 \%)$ \\
\hline \multicolumn{6}{|c|}{ Patients who had MRI } \\
\hline 1. $N=149$ & Clinical & $\begin{array}{c}107 \\
(71.8 \%)\end{array}$ & $23(15.4 \%)$ & $\begin{array}{c}42 \\
(28.2 \%)\end{array}$ & $19(12.8 \%)$ \\
\hline 2. $N=149$ & $\begin{array}{l}\text { Clinical plus } \\
\text { radiography }\end{array}$ & $\begin{array}{c}94 \\
(63.1 \%)\end{array}$ & $30(20.1 \%)$ & $\begin{array}{c}55 \\
(36.9 \%)\end{array}$ & $38(25.5 \%)$ \\
\hline 3. $N=149$ & $\begin{array}{c}\text { Clinical plus } \\
\text { radiography } \\
\text { plus MRI }\end{array}$ & $\begin{array}{c}70 \\
(\mathbf{4 7 . 0 \% )}\end{array}$ & $44(29.5 \%)$ & $\begin{array}{c}79 \\
(53.0 \%)\end{array}$ & $70(47.0 \%)$ \\
\hline
\end{tabular}


Table 2

\begin{tabular}{|c|c|c|c|}
\hline $\begin{array}{l}\text { Stage of global } \\
\text { assessment }\end{array}$ & Data source & $\begin{array}{l}\text { axSpA YES, } \\
\text { number (\%) }\end{array}$ & $\begin{array}{l}\text { axSpA NO, } \\
\text { number }(\%)\end{array}$ \\
\hline 2. $N=246$ & Clinical plus radiography & $141(57.3 \%)$ & $105(42.7 \%)$ \\
\hline 2. $N=246$ & $\begin{array}{l}\text { Clinical plus radiography after central } \\
\text { reader assessment }\end{array}$ & 79 (32.1\%) & $167(67.9 \%)$ \\
\hline 3. $N=149$ & Clinical plus radiography plus MRI & $70(47.0 \%)$ & 79 (53.0\%) \\
\hline 3. $N=149$ & $\begin{array}{l}\text { Clinical plus radiography plus MRI } \\
\text { after central reader assessment }\end{array}$ & 45 (30.2\%) & $104(69.8 \%)$ \\
\hline
\end{tabular}

(18.7\%), Crohn's colitis (31.3\%), ulcerative colitis (20.3\%). MRI was conducted in 149 patients. The number of patients diagnosed with axSpA by the local rheumatologist decreased after radiography and then decreased further after MRI while confidence in the diagnosis progressively increased (Table 1). After central evaluation of all patient data, the number of patients diagnosed with axSpA decreased substantially compared to assessment by local readers (Table 2).

Conclusion: In a setting of undiagnosed back pain and higher risk for axial $\mathrm{SpA}$, imaging is primarily helpful in ruling out SpA and reducing false positives. Despite this, central evaluation raises concerns regarding ascertainment of false positive SpA in routine practice.

Disclosure of Interests: Walter P Maksymowych Grant/research support from: AbbVie, Pfizer, Janssen, Novartis, Consultant for: AbbVie, Eli Lilly, Boehringer, Galapagos, Janssen, Novartis, Pfizer and UCB Pharma; Chief Medical Officer for Canadian Research and Education Arthritis, Raj Carmona Grant/research support from: Amgen, Abbvie, Jannsen, Consultant for: Amgen, Abbvie, BMS, Eli Lilly, Merck, Novartis, Jannsen, Takeda, UCB, James Yeung: None declared, Jon Chan Grant/research support from: Janssen, UCB, Novartis, Pfizer, Celgene, Consultant for: Amgen, Celgene, Eli Lilly, Janssen, Amgen, Abbvie, Novartis, Pfizer, UCB, Sandoz, Merck, Liam Martin: None declared, Sibel Aydin Consultant for: Abbvie, Celgene, UCB, Novartis, Jannsen, Sanofi, Dianne Mosher: None declared, Ariel Masetto Grant/research support from: Amgen, Sanofi, Consultant for: Sanofi, Pfizer, Bristol-Myers Squibb, Novartis, Boehringer Ingelheim, Speakers bureau: Novartis, Stephanie Keeling Consultant for: AbbVie. Pfizer, Eli Lily, Janssen, Amgen, Astrzeeneca, UCB., Olga Ziouzina: None declared, Sherry Rohekar Consultant for: Abbvie, Amgen, BMS, Celgene, Eli-Lilly, Janssen, Merck, Novartis, Pfizer, Roche, Sanofi, UCB, Joel Paschke: None declared, Amanda Carapellucci: None declared, Robert G Lambert Consultant for: Bioclinica, Parexel, Abbvie DOI:10.1136/annrheumdis-2019-eular.6080

\section{SAT0340 CHARACTERIZATION OF PATIENTS WITH AXIAL SPONDYLOARTHRITIS BY PRESENCE OF ENTHESITIS: DATA FROM THE CORRONA PSORIATIC ARTHRITIS/ SPONDYLOARTHRITIS (PSA/SPA) REGISTRY}

Philip J. Mease ${ }^{1}$, Mei Liu' ${ }^{2}$, Sabrina Rebello ${ }^{2}$, Winnie $\mathrm{Hua}^{2}$, Robert Mclean ${ }^{2}$, Esther $\mathrm{Yi}^{3,4}$, Yujin Park ${ }^{5}$, Alexis Ogdie ${ }^{6} .{ }^{1}$ Swedish Medical Center/Providence St. Joseph Health and University of Washington, Seattle, United States of America; ${ }^{2}$ Corrona, LLC, Waltham, United States of America; ${ }^{3}$ The University of Texas at Austin, Austin, United States of America; ${ }^{4}$ Baylor Scott and White Health, Temple, United States of America; ${ }^{5}$ Novartis Pharmaceuticals Corporation, East Hanover, United States of America; ${ }^{6}$ Perelman School of Medicine, University of Pennsy/vania, Philadelphia, United States of America

Background: Enthesitis is a common extra-axial manifestation in patients with axial spondyloarthritis $(A x S p A)^{1,2}$; however, not much is known about the prevalence of enthesitis in AxSpA and its impact on disease burden in US real-world settings.

Objectives: This study describes characteristics of patients with $\mathrm{AxSpA}$ who had enthesitis vs patients without enthesitis.

Methods: This study included patients aged $>18$ years with $\mathrm{AxSpA}$ enrolled in the Corrona PsA/SpA Registry between March 2013 and August 2018. Enthesitis at enrollment was assessed via the Spondyloarthritis Research Consortium of Canada (SPARCC) Enthesitis Index Patient demographics, clinical characteristics, treatment profiles, disease activity, quality of life, and work productivity were compared between patients with and without enthesitis using $t$ tests or Wilcoxon rank-sum tests for continuous variables and $\chi^{2}$ or Fisher's exact tests for categorical variables.

Results: Of 477 patients with AxSpA, 121 (25.4\%) had enthesitis. Symptom and disease duration were similar between patients with and without enthesitis. In patients with enthesitis, a higher proportion were female, were more likely to have non-radiographic $\operatorname{AxSpA}$ and a history of depression, serious infections, and fibromyalgia vs those without enthesitis (all $P<0.05$; Table 1). Current treatment with biologics or conventional
Table 1. Demographic and Clinical Characteristics and Treatment Profiles in Patients With AXSPA Stratified by Presence of Enthesitis at Enrollment

\begin{tabular}{|c|c|c|c|}
\hline Characteristic & $\begin{array}{l}\text { No Enthesitis } \\
(\mathrm{N}=356)\end{array}$ & $\begin{array}{l}\text { With Enthesitis } \\
(N=121)\end{array}$ & $P$ Value \\
\hline Non-radiographic AxSpA, $n(\%)$ & $56(15.7)$ & $34(28.1)$ & $<0.01$ \\
\hline Age, mean (SD) [n], years & $47.3(14.1)[351]$ & $47.3(13.1)[120]$ & 0.99 \\
\hline Female, $n / m(\%)$ & $112 / 351(31.9)$ & $61 / 121(50.4)$ & $<0.01$ \\
\hline White, $\mathrm{n} / \mathrm{m}(\%)$ & $319 / 344(92.7)$ & $105 / 119(88.2)$ & 0.16 \\
\hline Work status, $\mathrm{n}(\%)$ & $n=351$ & $n=121$ & 0.30 \\
\hline Full time & $214(61.0)$ & $69(57.0)$ & \\
\hline Part time & $22(6.3)$ & $7(5.8)$ & \\
\hline Disabled & $42(12.0)$ & $24(19.8)$ & \\
\hline Retired & $44(12.5)$ & $12(9.9)$ & \\
\hline Other & $29(8.3)$ & $9(7.4)$ & \\
\hline BMI, mean (SD) [n], kg/m² & $29.8(6.8)[345]$ & $29.9(6.9)[120]$ & 0.83 \\
\hline Symptom duration, mean (SD) [n], years & $16.8(11.8)[340]$ & $17.3(13.1)[118]$ & 0.67 \\
\hline Disease duration, mean (SD) [n]. years & $10.2(10.7)[347]$ & $8.2(10.3)[120]$ & 0.09 \\
\hline \multicolumn{4}{|l|}{ Comorbidities, $n(\%)$} \\
\hline Cardiovascular disease & $140(39.3)$ & $56(46.3)$ & 0.18 \\
\hline Hypertension & $109(30.6)$ & $37(30.6)$ & 0.99 \\
\hline Depression & $53(14.9)$ & $29(24.0)$ & 0.02 \\
\hline Hyperlipidemia & $52(14.6)$ & $21(17.4)$ & 0.47 \\
\hline Uveitis & $40(11.2)$ & $13(10.7)$ & 0.88 \\
\hline Metabolic syndrome & $27(7.6)$ & $9(7.4)$ & 0.96 \\
\hline Diabetes mellitus & $20(5.6)$ & $11(9.1)$ & 0.18 \\
\hline Psoriasis & $22(6.2)$ & $7(5.8)$ & 0.88 \\
\hline Serious infections & $16(4.5)$ & $12(9.9)$ & 0.03 \\
\hline Fibromyalgia & $11(3.1)$ & $11(9.1)$ & 0.01 \\
\hline Ulcerative colitis & $18(5.1)$ & $3(2.5)$ & 0.31 \\
\hline Any cancer (excluding NMSC) & $16(4.5)$ & $5(4.1)$ & 0.87 \\
\hline Crohn disease & $14(3.9)$ & $6(5.0)$ & 0.63 \\
\hline Prior csDMARD use, $n(\%)$ & $47(13.2)$ & $30(24.8)$ & $<0.01$ \\
\hline Prior biologic use, $\mathrm{n}(\%)$ & $97(27.2)$ & $47(38.8)$ & 0.02 \\
\hline Number of prior biologics, $n(\%)$ & & & 0.01 \\
\hline 0 & $259(72.8)$ & $74(61.2)$ & \\
\hline 1 & $68(19.1)$ & $26(21.5)$ & \\
\hline$\geq 2$ & $29(8.1)$ & $21(17.4)$ & \\
\hline Current biologic use, $\mathrm{n}(\%)$ & $237(66.6)$ & $84(69.4)$ & 0.56 \\
\hline $\begin{array}{l}\text { Current csDMARD use only (no biologics or } \\
\text { tsDMARDs), } n(\%)\end{array}$ & $30(8.4)$ & $12(9.9)$ & 0.62 \\
\hline Methotrexate (any), n (\%) & $37(10.4)$ & $21(17.4)$ & 0.04 \\
\hline
\end{tabular}

Table 2. Disease Activity, Quality of Life, and Work Productivity in Patients With AxSpA Stratified by Presence of Enthesitis at Enrollment

\begin{tabular}{|c|c|c|c|}
\hline Characteristic & $\begin{array}{l}\text { No Enthesitis } \\
(N=356)\end{array}$ & $\begin{array}{l}\text { With Enthesitis } \\
\qquad(\mathrm{N}=121)\end{array}$ & $P$ Value \\
\hline ASDAS, mean (SD) [n] & $2.5(1.2)[212]$ & $2.9(0.9)[79]$ & $<0.01$ \\
\hline $\operatorname{BASDAl}(0-10)$, mean $(\mathrm{SD})[\mathrm{n}]$ & $4.1(2.4)[340]$ & $5.3(2.2)[118]$ & $<0.01$ \\
\hline $\operatorname{BASFI}(0-10)$, mean (SD) [n] & $3.3(2.7)[338]$ & $4.5(2.8)[119]$ & $<0.01$ \\
\hline $\begin{array}{l}\text { Lateral lumbar flexion (average of left and } \\
\text { right), mean }(\mathrm{SD})[\mathrm{n}], \mathrm{cm}\end{array}$ & $21.5(18.8)[308]$ & $28.1(21.1)[116]$ & 0.01 \\
\hline Enthesitis, $n(\%)$ & - & $121(100.0)$ & - \\
\hline $\begin{array}{l}\text { SPARCC Enthesitis Index (1-16), mean } \\
\text { (SD) [n] }\end{array}$ & - & $3.9(2.9)$ [121] & - \\
\hline Dactylitis, n (\%) & $7(2.0)$ & $4(3.3)$ & 0.40 \\
\hline Dactylitis count (1-20), mean (SD) $[\mathrm{n}]$ & $3.6(3.9)[7]$ & $2.0(2.0)[4]$ & 0.35 \\
\hline Tender joint count $(0-68)$, mean $(\mathrm{SD})[\mathrm{n}]$ & $1.3(4.2)[347]$ & $8.2(10.8)[121]$ & $<0.01$ \\
\hline Swollen joint count $(0-66)$, mean (SD) [n] & $0.5(2.0)[347]$ & $1.3(3.3)[121]$ & $<0.01$ \\
\hline DAPSA, mean (SD) [n] & $15.6(8.3)[54]$ & $28.6(21.1)[12]$ & 0.02 \\
\hline DAPSA group, $n(\%)$ & $n=54$ & $n=12$ & 0.03 \\
\hline Remission & $2(3.7)$ & $1(8.3)$ & \\
\hline Low & $25(46.3)$ & $2(16.7)$ & \\
\hline Moderate & $21(38.9)$ & $4(33.3)$ & \\
\hline High & $6(11.1)$ & $5(41.7)$ & \\
\hline CDAPSA, mean $(\mathrm{SD})[\mathrm{n}]$ & $12.5(7.9)[109]$ & $22.1(18.1)[21]$ & 0.02 \\
\hline $\begin{array}{l}\text { Physician global assessment, mean (SD) } \\
\text { [n] }\end{array}$ & $23.5(21.9)[345]$ & $36.6(21.9)[117]$ & $<0.01$ \\
\hline $\begin{array}{l}\text { Physician global assessment of psoriasis, } \\
\text { mean (SD) [n] }\end{array}$ & $0.5(1.0)[70]$ & $0.1(0.5)[38]$ & 0.03 \\
\hline Patient pain (VAS 0-100), mean (SD) [n] & $43.5(29.5)[331]$ & $57.5(26.7)[111]$ & $<0.01$ \\
\hline Patient fatigue (VAS 0-100), mean (SD) [n] & $45(28.6)[353]$ & $56.5(27.5)[121]$ & $<0.01$ \\
\hline Morning stiffness, $n(\%)$ & $318(89.3)$ & $117(96.7)$ & 0.72 \\
\hline$<30$ minutes & $71(22.3)$ & $28(23.9)$ & \\
\hline$\geq 30$ minutes & $247(77.7)$ & $89(76.1)$ & \\
\hline $\begin{array}{l}\text { Patient global assessment (VAS 0-100), } \\
\text { mean (SD) [n] }\end{array}$ & $54.1(32.9)[115]$ & $49.8(29.7)[24]$ & 0.56 \\
\hline HAQ-DI (0-3), mean (SD) [n] & $0.6(0.6)[299]$ & $0.9(0.7)[89]$ & $<0.01$ \\
\hline EQ VAS $(0-100)$, mean $(S D)[n]$ & $66.4(22.0)[344]$ & $59.7(22.7)[120]$ & $<0.01$ \\
\hline \multicolumn{4}{|l|}{ WPAl domains, mean (SD) [n] } \\
\hline Current employment, $\mathrm{n} / \mathrm{m}(\%)$ & $241 / 351(68.7)$ & $75 / 119(63.0)$ & 0.26 \\
\hline$\%$ Work time missed & $5.8(16.8)[218]$ & $9.0(18.8)[71]$ & 0.06 \\
\hline$\%$ Impairment while working & $25.3(24.4)[230]$ & $38.3(27.4)[71]$ & $<0.01$ \\
\hline$\%$ Overall work impairment & $27.6(26.6)[210]$ & $41.4(29)[69]$ & $<0.01$ \\
\hline \% Activity impairment & $35.6(29.3)[343]$ & $49.8(29.5)[121]$ & $<0.01$ \\
\hline \multicolumn{4}{|c|}{$\begin{array}{l}\text { ASDAS, Ankylosing Spondylitis Disease Activity Score; AxSpA, axial spondyloarthritis; BASDAl, Bath } \\
\text { Ankylosing Spondylitis Disease Activity Index; BASFI, Bath Ankylosing Spondylitis Functional Index; } \\
\text { cDAPSA, Clinical Disease Activity Index for Psoriatic Arthritis; DAPSA, Disease Activity Index for Psoriatic } \\
\text { Arthritis; EQ VAS, EuroQol visual analogue scale; HAQ-DI, Health Assessment Questionnaire Disability } \\
\text { Index; SPARCC, Spondyloarthritis Research Consortium of Canada; VAS, visual analog scale; WPAI, Work } \\
\text { Productivity and Activity lmpairment Questionnaire. }\end{array}$} \\
\hline
\end{tabular}

synthetic disease-modifying antirheumatic drug (csDMARD) monotherapy was similar regardless of presence of enthesitis; however, patients with enthesitis were more likely to have prior csDMARD and biologic use, as well as current methotrexate use. Patients with enthesitis had worse disease activity (ASDAS, BASDAI, and BASFI scores; tender and swollen joint counts; physician global assessment; and DAPSA and CDAPSA scores), spinal mobility measures, quality of life (pain, fatigue, HAQ scores, and EQ VAS scores), and greater work impairment than patients without enthesitis (all $P<0.05$; Table 2).

Conclusion: Among patients with $\mathrm{AxSpA}$ in this US real-world study, the presence of enthesitis was associated with worse disease activity and quality of life compared to patients without enthesitis. 